\title{
TRIMODALITY THERAPY FOR STAGE II-III CARCINOMA OF THE ESOPHAGUS: A DOSE-RANGING STUDY OF CONCURRENT CAPECITABINE, DOCETAXEL, AND THORACIC RADIOTHERAPY
}

\author{
Matthew D. Wood`, Bassem I. Zaki, Stuart R. Gordon, John E. Sutton Jr., Mikhail Lisovsky, \\ Jiang Gui, Jeffrey A. Bubis, Konstantin H. Dragnev, and James R. Rigas \\ From the Comprehensive Thoracic Oncology Program, Norris Cotton Cancer Center, Dartmouth- \\ Hitchcock Medical Center, Lebanon, NH, and the Geisel School of Medicine at Dartmouth, \\ Hanover, $\mathrm{NH}$
}

\begin{abstract}
PURPOSE-This dose escalation study was performed to determine the recommended phase II dose of oral capecitabine to be delivered concurrently with thoracic radiation therapy and weekly docetaxel in patients with locally advanced esophageal carcinoma.

METHODS-Patients with operable stage II or III esophageal carcinoma were staged by endoscopic ultrasonography (EUS) and CT. Two cycles of docetaxel $\left(80 \mathrm{mg} / \mathrm{m}^{2}\right)$ and carboplatin (target area under the concentration curve $6 \mathrm{mg} * \mathrm{~min} / \mathrm{mL}$ ) were delivered over 6 weeks. This was followed by concurrent weekly docetaxel $\left(15 \mathrm{mg} / \mathrm{m}^{2}\right)$, thoracic radiotherapy $(50.4 \mathrm{~Gy}$ in 28 fractions), and increasing doses of capecitabine (500 $\mathrm{mg}$ to $3500 \mathrm{mg}$ ) given prior to each fraction of radiotherapy. Following re-staging, responding patients continued to esophagectomy within 4-8 weeks of completing chemoradiotherapy.
\end{abstract}

RESULTS-Forty-four patients (pts) were enrolled and 40 were evaluable for the dose-ranging component of concurrent chemoradiotherapy. EUS stages at enrollment were T3N1 (29 pts), T3N0 (4 pts), T2N1 (6 pts), and T4N0 (1 pts). The maximum tolerated dose of capecitabine was $3500 \mathrm{mg}$. Thirty-six patients had surgery; $83 \%$ had R0 resection and $17 \%$ had complete pathological response. Median overall survival was 23.5 months, with $34 \%$ and $27 \%$ alive at three and five years.

CONCLUSION-The recommended phase II dose of capecitabine is $3500 \mathrm{mg}$ when given concurrently with $50.4 \mathrm{~Gy}$ of thoracic radiotherapy in 28 fractions and weekly docetaxel. This trimodality therapy for operable locally advanced esophageal carcinoma was very well-tolerated and remarkably active. This regimen holds promise for treatment of esophageal carcinoma and warrants further investigation.

\section{INTRODUCTION}

Esophageal cancer is predicted to afflict 16,980 individuals in 2011, causing 14,710 deaths in that year. ${ }^{1}$ The disease has a poor outcome, with a $16.8 \%$ five-year survival rate, largely

\footnotetext{
Corresponding Author: James R. Rigas, M.D. Norris Cotton Cancer Center, Geisel School of Medicine at Dartmouth, One Medical Center Drive, Lebanon, New Hampshire 03756, Telephone: (603) 650-6344, Facsimile: (603) 650-9496, james.r.rigas@dartmouth.edu.

Current affiliation: The University of California San Francisco, Department of Pathology and Laboratory Medicine, San Francisco, CA.

Clinical Trials Registration Number: NCT00153881

Presented in part at the $40^{\text {th }}$ Annual Meeting of the American Society of Clinical Oncology, June 6, 2004, New Orleans, LA, USA, and reported at the $48^{\text {th }}$ Annual Meeting of the American Society of Clinical Oncology, June 1st, 2012, Chicago, IL, USA.
} 
because most patients present with regional or distant spread of disease. ${ }^{2}$ Surgical resection is a mainstay of treatment for stage II-III disease, but locoregional and systemic recurrence is common. Consequently, a number of clinical studies have focused on identifying Neoadjuvant therapies to prevent recurrence and improve survival.

Numerous studies have evaluated the role for Neoadjuvant chemotherapy and/or radiotherapy in surgically resectable esophageal carcinoma. ${ }^{3-5} \mathrm{~A}$ recent meta-analysis of Neoadjuvant therapy trials encompassing 4188 patients showed that the hazard ratio for allcause mortality was 0.78 for Neoadjuvant chemoradiotherapy and 0.87 for Neoadjuvant chemotherapy compared to surgery alone. ${ }^{6}$ As the use of Neoadjuvant chemoradiotherapy gained attention, a series of retrospective analyses indicated that addition of an intensive induction chemotherapy regimen prior to concomitant chemoradiotherapy and surgery further improves outcomes. ${ }^{7,8}$ Numerous phase II trials evaluating induction chemotherapy have shown that such regimens can be well-tolerated and highly active, but results have varied widely due to the variation in agents, schedules, and dosing. ${ }^{9-19}$ In the absence of an accepted optimal approach, one focus for current trials is to identify regimens which maximize quality of life and have minimal toxicity while maintaining acceptable clinical response and overall survival.

The pyrimidine analog 5-fluorouracil (5-FU) is one of the most commonly studied chemotherapeutic agents in esophageal cancer. ${ }^{20}$ This agent requires central venous access for delivery, which carries a risk of infection, as well as being inconvenient for patients. Capecitabine is an orally bioavailable prodrug of $5^{\prime}$-deoxy-5-fluorouridine (5-DFUR), which is converted to 5-FU in tumor cells due to high expression of the converting enzyme thymidine phosphorylase in human carcinomas. ${ }^{21}$ Capecitabine has shown promise in phase I and phase II studies for the treatment of esophageal cancer. ${ }^{22-25}$ High expression of thymidine phosphorylase and was associated with therapeutic response to capecitabine/ cisplatin combination therapy in patients with esophageal carcinoma, indicating that capecitabine may be a valuable agent for esophageal cancer treatment due to tumor responsiveness and lower general toxicity. ${ }^{26}$

Docetaxel and paclitaxel are taxane class agents that stabilize microtubules and interfere with cell division. Both agents have a broad spectrum of antitumor activity, and both have been studied in esophageal cancer. ${ }^{27-29}$ Taxane treatment increases expression of thymidine phosphorylase in mouse model systems of breast and colorectal cancer. ${ }^{30,31}$ Therefore, treatment regimens containing docetaxel and capecitabine may have high therapeutic value due to a synergistic effect between these two agents. Preliminary studies of combination capecitabine/docetaxel showed that these agents are well-tolerated and highly active, but the study was limited by a small sample size and limited follow-up. ${ }^{32}$ The goal of this trial was to integrate oral capecitabine into a trimodal therapeutic regimen including induction chemotherapy followed by combination chemoradiotherapy and surgical resection. Capecitabine dosing was titrated upward during chemoradiotherapy, and patients were monitored for the primary outcome of dose-limiting toxicity. Secondary outcomes were overall survival, relapse-free survival, the rate of complete pathological response, and the rate complete surgical resection.

\section{METHODS}

\section{Patient Selection}

Previously untreated patients with locally advanced, surgically resectable cancer of the esophagus or gastroesophageal (GE) junction, clinical stage II-III (T2-4N0M0, T1-4N1M0) with histologic or cytologic evidence of adenocarcinoma or squamous cell carcinoma confirmed by the Department of Pathology at Dartmouth-Hitchcock Medical Center were 
eligible. Patients having gastric cancer with minor involvement of the GE junction or distal esophagus were not eligible, nor were patients with cancer of the cervical esophagus. Celiac lymph node metastases were considered regional lymph node involvement (N1). Patients were required to be $\geq 18$ years of age, with Karnofsky performance status $\geq 70 \%$, and with adequate organ function defined by leukocytes $\geq 3,000 / \mathrm{mm}^{3}$, platelets $\geq 100,000 / \mathrm{mm}^{3}$, bilirubin $\leq$ to the upper limit of normal (ULN), serum transaminase (AST or ALT) $\leq$ to five times the ULN, serum creatinine $\leq 1.5 \mathrm{mg} / \mathrm{dL}$ or creatinine clearance of $50 \mathrm{mg} * \mathrm{~min} / \mathrm{dL}$. Patients were not permitted to have prior chemotherapy, immunotherapy, or chest/ abdominal radiotherapy. Patients with any of the following were excluded: clinically apparent metastatic disease (brain, bone, pulmonary, or liver metastases, or positive cytology of the pleura, pericardium, or peritoneum), clinically evident metastatic disease of the cervical or supraclavicular lymph nodes, grade $\geq 2$ peripheral neuropathy, serum calcium $>12 \mathrm{mg} / \mathrm{dL}$, recurrent laryngeal or phrenic nerve paralysis, tracheobronchial tree involvement, tracheoesophageal fistula, concurrent active malignancy other than nonmelanoma skin cancer or carcinoma-in-situ of the cervix, or uncontrolled intercurrent medical illness. The study was performed in accordance with the Committee for the Protection of Human Subjects (CPHS) at Dartmouth College and monitored by the Data Monitoring Committee of the Norris Cotton Cancer Center at Geisel School of Medicine at Dartmouth. All patients signed a CPHS-approved informed consent prior to enrollment.

\section{Clinical Staging}

Initial evaluation included CT scans of the chest and upper abdomen, upper GI endoscopy with endoscopic ultrasonography (EUS), laboratory tests, and radiation therapy simulation, all completed within four weeks of enrollment. A bone scan was performed in patients with abnormal alkaline phosphatase levels in the setting of normal AST, or patients with newonset bone pain. A screening MRI or CT scan of the brain was performed for a recent history of headache or new focal neurologic findings. EUS was performed with an Olympus gastroscope under conscious sedation in the usual fashion. Biopsies were taken at the initial clinical staging and fixed for evaluation by Pathology. Prior to surgical resection, patients were re-staged by repeat CT scans of the chest and upper abdomen and upper GI endoscopy with EUS.

\section{Treatment Plan}

In the induction phase, patients were treated with two cycles of docetaxel $\left(80 \mathrm{mg} / \mathrm{m}^{2}\right)$ and carboplatin (target area under the concentration curve (AUC) of $6 \mathrm{mg} * \mathrm{~min} / \mathrm{mL}$ ) every 21 days for six weeks. In the concurrent chemoradiotherapy (CCRT) phase, all patients received weekly docetaxel $\left(15 \mathrm{mg} / \mathrm{m}^{2}\right)$ and external beam radiotherapy (50.4 Gy at 1.8 Gy per fraction in 28 fractions, delivered Monday through Friday). The patients underwent CT simulation, immobilized, arms up using a wing-board combined with a vac-bag. All patients underwent 3 dimensional treatment planning. The dose was calculated using tissue heterogeneity corrections. The planned treatment volume 1 (PTV1) included the gross tumor volume (GTV) with a 4-5 cm margin proximally, distally and $1.5-2 \mathrm{~cm}$ circumferentially. The celiac nodes were electively covered in distal and gastro-esophageal junction carcinomas. The planned treatment volume 2 (PTV2) was designed with a $1.5-2 \mathrm{~cm}$ margin around the GTV. Margins around the grossly positive nodes were kept at $1-1.5 \mathrm{~cm}$ in both PTV1 and PTV2. PTV 1 received 41.4 Gy in 23 fractions. A boost of 9 Gy in 5 fractions was delivered to PTV2. The constraints for the organs at risk were kept under tolerance: less than $32 \%$ of the lung volumes received more than $20 \mathrm{~Gy}$, less than $50 \%$ of the heart volume received more than $40 \mathrm{~Gy}$, and the maximum dose to any point on the spinal cord was kept less than $45 \mathrm{~Gy}$. Prior to each fraction of radiotherapy, patients took a set dose of oral capecitabine ranging from $500 \mathrm{mg}$ at dose level I to $3500 \mathrm{mg}$ at dose level VII, in $500 \mathrm{mg}$ increments. Dose-limiting toxicity (DLT) was defined as any grade 3 or higher toxicity 
which did not return to grade 2 or lower within three weeks from completion of treatment, or any grade toxicity which resulted in a treatment break longer than one week. Initially, three patients were enrolled at dose level I. If one or more patients developed DLT, three more patients were enrolled at the same dose level. If there was no DLT, the next three patients were enrolled at the next incremental dose level. The maximum tolerated dose (MTD) was defined as the dose of capecitabine at which $33 \%$ of patients experience a DLT. This experimental design follows the approach outlined by Storer for dose-limiting toxicity studies. ${ }^{33}$

\section{Efficacy Analysis}

The primary outcome for this study is the occurrence of dose-limiting toxicity during the escalation phase of concomitant chemoradiotherapy treatment. Secondary outcomes include changes in patient weight and performance status after chemoradiotherapy, frequency of minor toxicity, overall survival, relapse-free survival, disease-specific survival, curative surgical resection rate, and pathological complete response rate. All survival outcomes were determined from the date of study enrollment. Relapse-free survival (RFS) was measured to the date of documented disease progression, incomplete surgical resection, or death from any cause. Overall survival (OS) was measured to the date of death from any cause, regardless of disease status. Disease-specific survival (DSS) was measured to the date of death from esophageal cancer or complications of surgery, with other causes of death censored at the time of death. Patients who were living or lost to follow-up without progressive disease were censored at the date of the last documented clinical encounter. Surgical outcomes were defined as: R0, curative resection, all gross disease removed, surgical margins free of tumor; R1, palliative resection, gross tumor left behind, or positive surgical margins on pathology; or R2, no resection, primary tumor could not be removed. Positive radial margins were considered R1 resection. Pathological complete response (pCR) was defined as the absence of residual carcinoma at the primary site on microscopic examination. Microscopic residual disease (MRD) was defined as only single cancer cells or groups of cancer cells present, according to the reporting pathologist. Significant residual disease was defined as residual carcinoma present in the background of fibrosis or as no tumor kill present. All cases that were downstaged to ypT0 or ypT1 had slides reviewed by a pathologist for pCR or MRD, regardless of nodal or distant disease. Surgical mortality was defined as death within 30 days of the surgical procedure.

\section{RESULTS}

\section{Patient Characteristics}

Forty-four patients were enrolled. Four patients were replaced during the induction phase two due to discovery of metastatic disease during staging, one due to withdrawal of consent prior to treatment, and one due to death from a non-neoplastic cause (CVA). Demographic and clinical characteristics of the 40 patients who initiated induction are summarized in Table 1. Patients had a median age of 63 years (range 47 to 87 years), 32 (80\%) were male, and $36(90 \%)$ had adenocarcinoma. Clinical stages at enrollment were T3N1 $(\mathrm{N}=29,72.5 \%)$, T2N1 $(\mathrm{N}=6,15 \%), \mathrm{T} 3 \mathrm{~N} 0(\mathrm{~N}=410 \%)$, and T4N0 $(\mathrm{N}=1,2.5 \%)$ with disease involving the distal esophagus $(\mathrm{N}=12,30 \%)$, the distal esophagus and the GE junction $(\mathrm{N}=19,47.5 \%)$, or both these areas and the cardia of the stomach $(\mathrm{N}=8,20 \%)$. One patient $(2.5 \%)$ had disease of the mid-esophagus. The accrual and treatment course of the cohort is summarized in Figure 1. During the concomitant chemoradiotherapeutic (CCRT) phase, one patient died from ARDS, while two patients withdrew consent for low-grade toxicity. Both of these patients later continued to surgery: one had surgical resection, while the other was found to have liver metastases, so no resection was performed. Of 37 patients who completed CCRT, one declined surgery and one transitioned to hospice care, while the remaining 35 patients 
proceeded to surgery. The total number of patients having resection was therefore 36 ( $90 \%$ of those who received induction), one of whom did not complete the full course of CCRT. Thirty-three of 36 patients survived the surgical and post-operative period, for a surgical mortality rate of $8.3 \%$. One patient was found to have stage IV disease at the time of surgery.

\section{Safety Parameters}

There were seven dose limiting toxicities in CCRT phase, occurring across five different doses of capecitabine (Table 2). Two patients had grade 3 dysphagia (at $500 \mathrm{mg}$ and 1500 $\mathrm{mg}$ capecitabine), one patient had thrombocytopenia (at $1500 \mathrm{mg}$ ), one had fatigue (at 3000 $\mathrm{mg}$ ), and one had grade 3 pneumonitis (at $3500 \mathrm{mg}$ ). Two patients at $3500 \mathrm{mg}$ withdrew consent for toxicities - one for severe nausea and one for persistent neuropathy. Although these were objectively less than grade 3 or 4 events, the patients were unable to tolerate these toxic effects and declined further participation in the study. Because of the clinical impact of these events and the associated prolonged treatment interruption, we considered them to be dose-limiting. Therefore, from a total of nine patients treated at $3500 \mathrm{mg}$, three patients $(33.3 \%)$ had dose-limiting toxicity, meeting the definition for maximally tolerated dose.

All grade 3 or 4 toxicities (including the dose-limiting toxicities) which occurred among patients receiving CCRT are enumerated in Table 3. The most common non-hematologic event was dysphagia, occurring in 6 patients (15\%). The most common hematologic toxicity was ANC abnormality during induction, occurring in 10 patients (25\%). More than half of the participants had no events of grade 3 or higher $(\mathrm{N}=23,58 \%)$, and several categories of adverse event never occurred with severity above grade 2 .

\section{Efficacy parameters}

The median follow-up time was 18.5 months. Overall and relapse-free survivals were computed from 40 patients who started induction therapy, measured from the time of enrollment (Figure 2). The median overall survival was 23.5 months, with $62 \%, 34 \%$, and $27 \%$ of patients alive at one, three, and five years of follow-up. The median relapse-free survival was 15.6 months, with $63 \%$ of patients relapse-free at one year. The three- and fiveyear relapse-free survivals were both $37 \%$. The median disease-specific survival was 28.1 months (Figure 3A).

Thirty-six patients had surgical resection, with the vast majority (30 out of 36, 83\%) having $\mathrm{R} 0$ resection. One of the patients with $\mathrm{R} 0$ resection of the primary tumor was found to have a single nodule of metastatic disease embedded in the abdominal wall, which was completely resected. Five patients had R1 resection, with tumor microscopically present at the proximal, distal, or radial margins. Two of these patients had positive radial margins and two others had isolated tumor cells in lymphovascular spaces. Only one patient had microscopic extension of the primary tumor to a proximal or distal margin. One patient had gross residual disease involving the chest wall, and was classified as R2. Six patients $(16.7 \%, 95 \%$ confidence interval $4.5 \%-28.8 \%)$ had a complete pathological response (pCR) at the primary site, and an additional five patients had microscopic foci of residual tumor cells at the primary site. Based on evidence that microscopically residual disease (MRD) and complete pathological response have identical outcomes with respect to overall survival, we considered these two groups together. ${ }^{34} \mathrm{We}$ found that 11 patients $(30.6 \%, 95 \%$ confidence interval $15.5 \%-45.6 \%$ ) who had pCR or MRD had a median survival of 86.6 months, compared to a median survival of 19.1 months for 25 patients who had a greater extend of residual disease. The pCR/MRD group had a $61.4 \%$ survival rate at both 3 and 5 years, compared to $27.5 \%$ at 3 years and $18.4 \%$ at 5 years for patients with significant 
residual disease (Figure 3B). This result was statistically significant by the log-rank test, with a p-value of 0.026 .

Nineteen of 37 patients who completed CCRT (51\%) had a stable or improved Karnofsky performance status, while 13 patients (35\%) dropped by ten points and five patients (14\%) dropped by twenty points. No patient dropped their performance status dropped below $70 \%$. The average weight loss among CCRT patients was $4.6 \mathrm{~kg}$. Six patients (15\%) required a feeding tube prior to surgery, and in 3 of these cases the feeding tube was required because of the tumor causing obstruction of the esophagus rather than toxicity from the chemoradiotherapy.

\section{Discussion}

Esophageal cancer presents a therapeutic challenge due to the advanced stage of disease at clinical presentation, and frequent local recurrence. One approach to therapy consists of an intensive chemotherapeutic regimen, followed by lower doses of chemotherapy combined with radiotherapy. This approach may improve local disease control, achieve downstaging for easier surgical resection, and treat occult metastatic disease. However, such regimens can carry significant toxicity. Capecitabine and docetaxel are promising agents for such trimodal therapy, due to potential synergistic effects, reduced toxicity, and easy delivery. We investigated a trimodal regimen including phase 2 dose escalation of capecitabine in patients with resectable esophageal cancer and found that this regimen produced excellent median and five year survival, with a low burden of toxicity.

Several recent phase 2 studies have evaluated trimodal therapies in esophageal carcinoma, included both adenomatous and squamous histology, with a high-intensity induction chemotherapy phase before concomitant chemoradiotherapy (Table 4). The rates of R0 resections have ranged from $76 \%$ to $100 \%$. By comparison, our study achieved a reasonable rate of $83 \% \mathrm{R} 0$ resection. In four out of five $\mathrm{R} 1$ resections, disease extended to the radial margins or to the lyphovascular space, while only one case had a microscopically positive margin at the proximal or distal aspect. Thus, even in cases that were strictly defined as R1, the degree of marginal disease was minimal, suggesting a potent therapeutic effect. In the same set of studies, pathologic complete response rates among patients who had resection ranged from $20 \%$ to $40 \%$, while our study achieved a pCR rate of $17 \%$ and $\mathrm{pCR} / \mathrm{MRD}$ rate of $30.6 \%$. This may be a result of the sample size, and in fact the $95 \%$ confidence interval for our response rate overlaps with many of the response rates in previous studies. This may reflect the range of efficacy for this trimodal regimen, it must be noted that our surgical outcomes are confounded by the dose escalation aspect of the chemoradiotherapy phase. It is possible that using the maximum tolerated dose of capecitabine $(3500 \mathrm{mg}$ ) would produce better and less variable results. Furthermore, recent reports have found that tumor histology can influence treatment response. Specifically, patients with squamous cell carcinoma are more likely to achieve a pCR, but they are also more prone to distal recurrence. ${ }^{35}$ Thus, one factor potentially explaining our lower than expected $\mathrm{pCR}$ rate may be a higher frequency of adenocarcinoma compared to most other studies (Table 4). In this cohort, 34 patients (85\%) had T3 or higher staging at enrollment, which is comparable to other studies. Compared to a landmark clinical trial of 5-FU and cisplatin, this regimen produced a superior median overall survival (23.5 months, versus 18 months) and three-year survival ( $34 \%$ compared to $25 \%) .{ }^{36}$

As expected, we observed that patients having either pCR or MRD had a better 5-year survival compared to patients with a greater burden of residual disease. Although there is evidence that these two categories can be considered together, Verlato et al recently reported that the prognostic value of absent or scattered residual cancer cells varies depending on 
node status. ${ }^{37}$ Considering that the main goal of this work is to define maximum tolerated dose of capecitabine, and consequently we have a small number of patients in the cohort, we do not believe it is appropriate to draw any firm conclusions on the prognostic value of $\mathrm{pCR}$ versus MRD in our population.

One advantage of this regimen is the overall tolerability. Grade 3 or 4 toxicity occurred in a minority of patients, and was largely limited to dysphagia, nausea/vomiting, and laboratory abnormalities. Few patients required a feeding tube prior to surgery, indicating that induction therapy helped to preserve swallowing function, which enabled delivery of oral capecitabine. These results are consistent with data from a smaller set of patients who were treated with a capecitabine/docetaxel-based regimen. ${ }^{32}$ One limitation of our study is that at the highest tolerated dose level of capecitabine -- $3500 \mathrm{mg}$-- two of the patients had objectively low-grade toxicities which resulted in withdrawal from the study. It is possible that higher doses of capecitabine might be tolerated without producing grade 3 or higher toxicities, but our study was limited by our definition of DLT to include prolonged treatment breaks. We concluded that $3500 \mathrm{mg}$ capecitabine was the highest practically achievable dose, and an appropriate end point for a MTD study.

In conclusion, a trimodal approach including capecitabine and docetaxel was well-tolerated and produced acceptable overall survival rates, although the pathological response rate was low compared to other studies. The low pCR rate was a surprising result, but we believe the high tolerability and favorable survival outcomes make a strong case for further studies with the maximum tolerated dose of capecitabine, where better pCR rates might be attained. Despite the low pCR rate, overall survival was comparable to other studies and this is a more direct outcome. We identified $3500 \mathrm{mg}$ as the maximum tolerated dose of capecitabine in this regimen. We suggest that survival and response might be further improved by treating with the maximum tolerated capecitabine dose. High-grade toxicity was uncommon. This method of treatment warrants further investigation and has the potential to prolong survival and increase quality of life among patients with resectable esophageal carcinoma.

\section{Acknowledgments}

Funding provided by Sanofi-Aventis and National Institute of Health, 5P30 CA023108-33. Capecitabine supplied by Hoffmann-La Roche.

\section{References}

1. Siegel R, Ward E, Brawley O, et al. Cancer statistics, 2011: the impact of eliminating socioeconomic and racial disparities on premature cancer deaths. CA Cancer J Clin. 2011; 61:21236. [PubMed: 21685461]

2. Esophagus. SEER Stat Fact Sheets. Update 11/10/2011

3. Walsh TN, Noonan N, Hollywood D, et al. A comparison of multimodal therapy and surgery for esophageal adenocarcinoma. N Engl J Med. 1996; 335:462-7. [PubMed: 8672151]

4. Forastiere AA, Orringer MB, Perez-Tamayo C, et al. Concurrent chemotherapy and radiation therapy followed by transhiatal esophagectomy for local-regional cancer of the esophagus. J Clin Oncol. 1990; 8:119-27. [PubMed: 2295902]

5. Urba SG, Orringer MB, Turrisi A, et al. Randomized trial of preoperative chemoradiation versus surgery alone in patients with locoregional esophageal carcinoma. J Clin Oncol. 2001; 19:305-13. [PubMed: 11208820]

6. Sjoquist KM, Burmeister BH, Smithers BM, et al. Survival after neoadjuvant chemotherapy or chemoradiotherapy for resectable oesophageal carcinoma: an updated meta-analysis. Lancet Oncol. 2011; 12:681-92. [PubMed: 21684205] 
7. Jin J, Liao Z, Zhang Z, et al. Induction chemotherapy improved outcomes of patients with resectable esophageal cancer who received chemoradiotherapy followed by surgery. Int J Radiat Oncol Biol Phys. 2004; 60:427-36. [PubMed: 15380576]

8. Malaisrie SC, Hofstetter WL, Correa AM, et al. The addition of induction chemotherapy to preoperative, concurrent chemoradiotherapy improves tumor response in patients with esophageal adenocarcinoma. Cancer. 2006; 107:967-74. [PubMed: 16874819]

9. Bains MS, Stojadinovic A, Minsky B, et al. A phase II trial of preoperative combined-modality therapy for localized esophageal carcinoma: initial results. J Thorac Cardiovasc Surg. 2002; 124:270-7. [PubMed: 12167786]

10. De Vita F, Orditura M, Martinelli E, et al. A multicenter phase II study of induction chemotherapy with FOLFOX-4 and cetuximab followed by radiation and cetuximab in locally advanced oesophageal cancer. Br J Cancer. 2011; 104:427-32. [PubMed: 21245865]

11. Ruhstaller T, Widmer L, Schuller JC, et al. Multicenter phase II trial of preoperative induction chemotherapy followed by chemoradiation with docetaxel and cisplatin for locally advanced esophageal carcinoma (SAKK 75/02). Ann Oncol. 2009; 20:1522-8. [PubMed: 19465425]

12. Ruhstaller T, Pless M, Dietrich D, et al. Cetuximab in combination with chemoradiotherapy before surgery in patients with resectable, locally advanced esophageal carcinoma: a prospective, multicenter phase IB/II Trial (SAKK 75/06). J Clin Oncol. 2011; 29:626-31. [PubMed: 21205757]

13. Ajani JA, Komaki R, Putnam JB, et al. A three-step strategy of induction chemotherapy then chemoradiation followed by surgery in patients with potentially resectable carcinoma of the esophagus or gastroesophageal junction. Cancer. 2001; 92:279-86. [PubMed: 11466680]

14. Ajani JA, Walsh G, Komaki R, et al. Preoperative induction of CPT-11 and cisplatin chemotherapy followed by chemoradiotherapy in patients with locoregional carcinoma of the esophagus or gastroesophageal junction. Cancer. 2004; 100:2347-54. [PubMed: 15160337]

15. Ajani JA, Correa AM, Walsh GL, et al. Trimodality therapy without a platinum compound for localized carcinoma of the esophagus and gastroesophageal junction. Cancer. 2010; 116:1656-63. [PubMed: 20143431]

16. Henry LR, Goldberg M, Scott W, et al. Induction cisplatin and paclitaxel followed by combination chemoradiotherapy with 5-fluorouracil, cisplatin, and paclitaxel before resection in localized esophageal cancer: a phase II report. Ann Surg Oncol. 2006; 13:214-20. [PubMed: 16418887]

17. Swisher SG, Ajani JA, Komaki R, et al. Long-term outcome of phase II trial evaluating chemotherapy, chemoradiotherapy, and surgery for locoregionally advanced esophageal cancer. Int J Radiat Oncol Biol Phys. 2003; 57:120-7. [PubMed: 12909224]

18. Choong NW, Mauer AM, Haraf DC, et al. Long-term outcome of a phase II study of docetaxelbased multimodality chemoradiotherapy for locally advanced carcinoma of the esophagus or gastroesophageal junction. Med Oncol. 2011; 28 (Suppl 1):152-61.

19. Eisterer W, Vries DEA, Kendler D, et al. Triple Induction Chemotherapy and Chemoradiotherapy for Locally Advanced Esophageal Cancer. A Phase II Study. Anticancer Res. 2011; 31:4407-12. [PubMed: 22199307]

20. Kelsen DP, Ilson DH. Chemotherapy and combined-modality therapy for esophageal cancer. Chest. 1995; 107:224S-232S. [PubMed: 7781398]

21. Dooley M, Goa KL. Capecitabine. Drugs. 1999; 58:69-76. discussion 77-8. [PubMed: 10439930]

22. Koo DH, Park SI, Kim YH, et al. Phase II study of use of a single cycle of induction chemotherapy and concurrent chemoradiotherapy containing capecitabine/cisplatin followed by surgery for patients with resectable esophageal squamous cell carcinoma: long-term follow-up data. Cancer Chemother Pharmacol. 2011

23. Yun T, Han JY, Lee JS, et al. Phase II study of weekly paclitaxel and capecitabine in patients with metastatic or recurrent esophageal squamous cell carcinoma. BMC Cancer. 2011; 11:385. [PubMed: 21888637]

24. Cunningham D, Okines AF, Ashley S. Capecitabine and oxaliplatin for advanced esophagogastric cancer. N Engl J Med. 2010; 362:858-9. [PubMed: 20200397]

25. Spigel DR, Greco FA, Meluch AA, et al. Phase I/II trial of preoperative oxaliplatin, docetaxel, and capecitabine with concurrent radiation therapy in localized carcinoma of the esophagus or gastroesophageal junction. J Clin Oncol. 2010; 28:2213-9. [PubMed: 20351330] 
26. Lee S, Park YH, Kim KH, et al. Thymidine synthase, thymidine phosphorylase, and excision repair cross-complementation group 1 expression as predictive markers of capecitabine plus cisplatin chemotherapy as first-line treatment for patients with advanced oesophageal squamous cell carcinoma. Br J Cancer. 2010; 103:845-51. [PubMed: 20700125]

27. Ajani JA, Moiseyenko VM, Tjulandin S, et al. Clinical benefit with docetaxel plus fluorouracil and cisplatin compared with cisplatin and fluorouracil in a phase III trial of advanced gastric or gastroesophageal cancer adenocarcinoma: the V-325 Study Group. J Clin Oncol. 2007; 25:3205-9. [PubMed: 17664467]

28. Ajani JA, Moiseyenko VM, Tjulandin S, et al. Quality of life with docetaxel plus cisplatin and fluorouracil compared with cisplatin and fluorouracil from a phase III trial for advanced gastric or gastroesophageal adenocarcinoma: the V-325 Study Group. J Clin Oncol. 2007; 25:3210-6. [PubMed: 17664468]

29. Lorenzen S, Duyster J, Lersch C, et al. Capecitabine plus docetaxel every 3 weeks in first- and second-line metastatic oesophageal cancer: final results of a phase II trial. Br J Cancer. 2005; 92:2129-33. [PubMed: 15942631]

30. Endo M, Shinbori N, Fukase Y, et al. Induction of thymidine phosphorylase expression and enhancement of efficacy of capecitabine or $5^{\prime}$-deoxy-5-fluorouridine by cyclophosphamide in mammary tumor models. Int J Cancer. 1999; 83:127-34. [PubMed: 10449619]

31. Sawada N, Ishikawa T, Fukase Y, et al. Induction of thymidine phosphorylase activity and enhancement of capecitabine efficacy by taxol/taxotere in human cancer xenografts. Clin Cancer Res. 1998; 4:1013-9. [PubMed: 9563897]

32. Rao S, Oelschlager B, Koh WJ, et al. Phase I trial with weekly docetaxel, capecitabine and carboplatin followed by concomitant capecitabine and radiation in patients with locally advanced esophageal cancer. Journal of Clinical Oncology. 2008

33. Storer BE. Design and analysis of phase I clinical trials. Biometrics. 1989; 45:925-37. [PubMed: 2790129]

34. Mandard AM, Dalibard F, Mandard JC, et al. Pathologic assessment of tumor regression after preoperative chemoradiotherapy of esophageal carcinoma. Clinicopathologic correlations. Cancer. 1994; 73:2680-6. [PubMed: 8194005]

35. Koshy M, Greenwald BD, Hausner P, et al. Outcomes after trimodality therapy for esophageal cancer: the impact of histology on failure patterns. Am J Clin Oncol. 2011; 34:259-64. [PubMed: 20686405]

36. Kelsen DP, Ginsberg R, Pajak TF, et al. Chemotherapy followed by surgery compared with surgery alone for localized esophageal cancer. N Engl J Med. 1998; 339:1979-84. [PubMed: 9869669]

37. Verlato G, Zanoni A, Tomezzoli A, et al. Response to induction therapy in oesophageal and cardia carcinoma using Mandard tumour regression grade or size of residual foci. Br J Surg. 2010; 97:719-25. [PubMed: 20306529] 


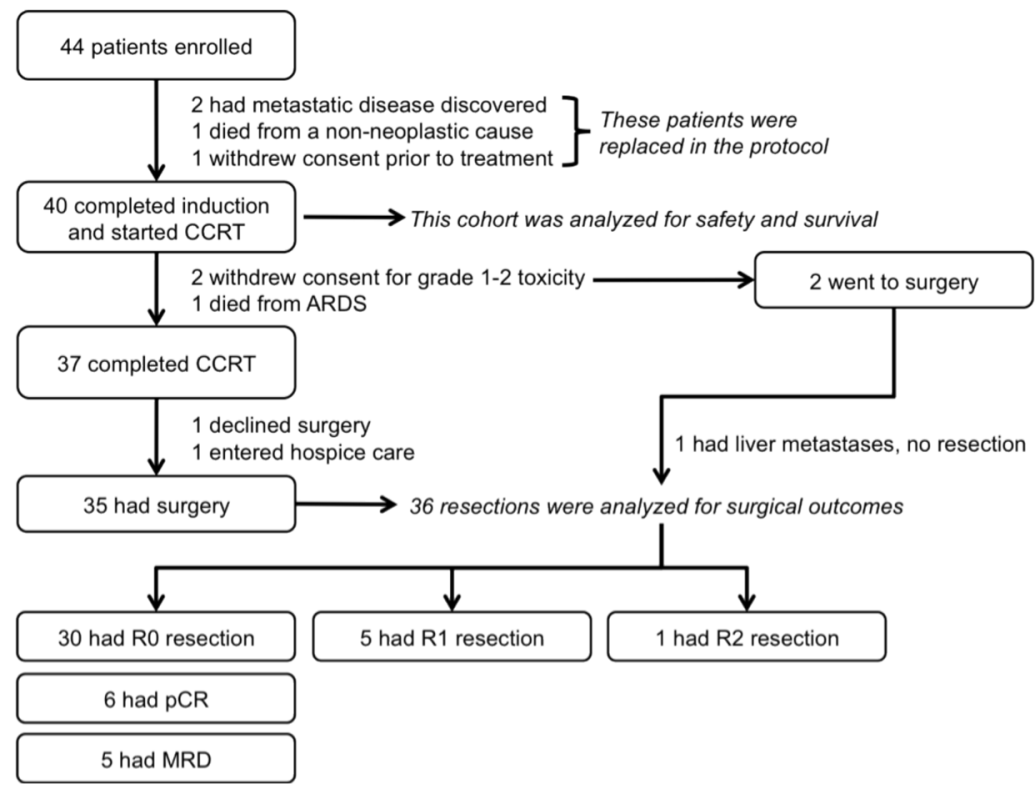

Figure 1. Accrual and Treatment Course

Patients accrued and treated throughout the trial are shown. CRT = chemoradiotherapy, $\mathrm{pCR}$ $=$ pathological complete response, $\mathrm{MRD}=$ microscopically residual disease . 
A.

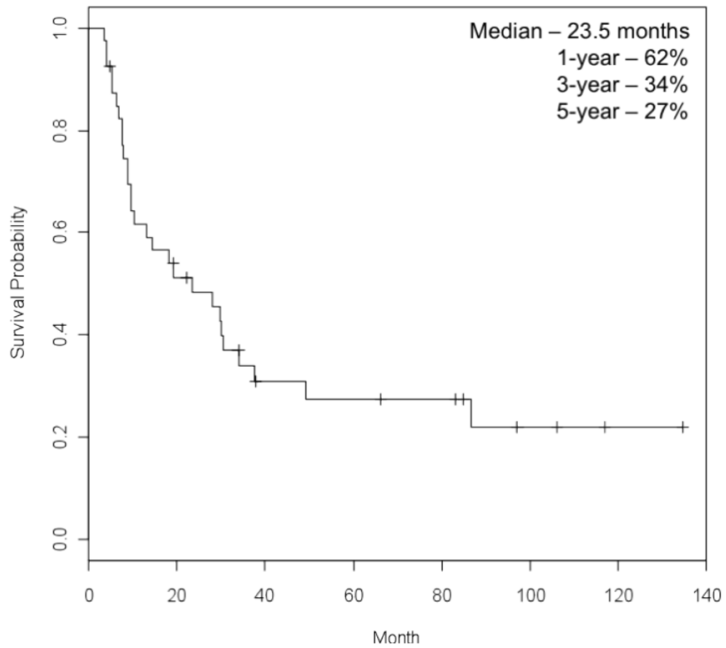

B.

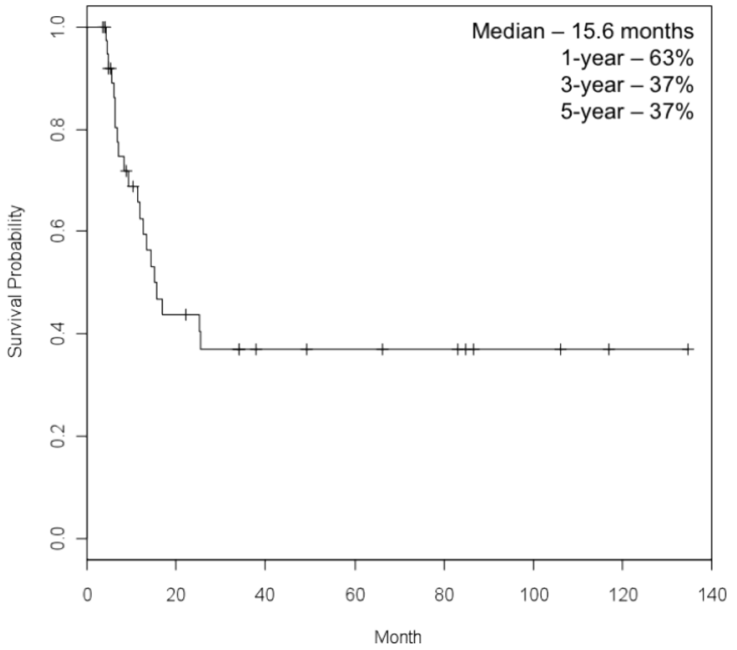

Figure 2. Overall and Relapse-Free Survival

Kaplan Meier curves are shown for overall survival (A) and relapse-free survival (B) among 40 patients who initiated induction therapy. Values for median overall survival and absolute survival at one, three, and five years are indicated on each chart. 

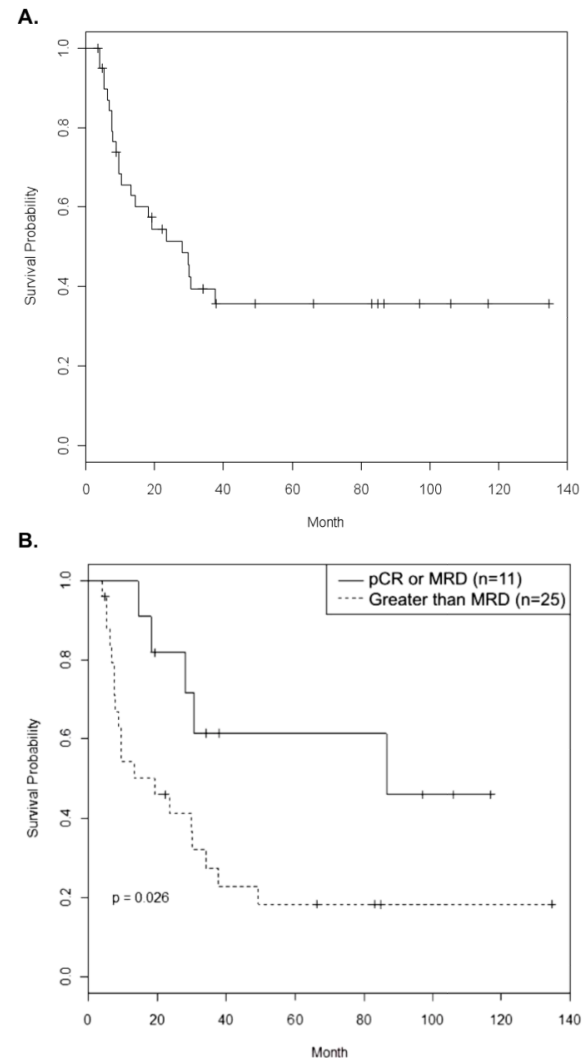

Figure 3. Disease-Specific and Subgroup Survival

(A) Disease-specific survival was calculated among enrolled patients. (B) Kaplan Meier curves for overall survival among 36 patients who had surgical resection are shown for patients having complete pathological response or microscopically residual disease $(n=11)$ versus those having a higher level of residual disease $(n=25)$. The $p$-value represents a logrank test. 
Table 1

Patient Characteristics

\begin{tabular}{|l|c|c|}
\hline & Number & Percent \\
\hline Patients & 40 & \\
\hline Age (years) & & \\
Median & 63 & \\
Range & $47-87$ & \\
\hline Sex & & \\
Male & 32 & $80 \%$ \\
Female & 8 & $20 \%$ \\
Performance Status & & \\
$90 \%$ & 29 & $72.5 \%$ \\
$80 \%$ & 8 & $20 \%$ \\
$70 \%$ & 3 & $7.5 \%$ \\
\hline Sites of Disease & 12 & $30 \%$ \\
Distal esophagus (DE) & 19 & $47.5 \%$ \\
DE and GE junction & 8 & $20 \%$ \\
DE, GE junction, and cardia & 1 & $2.5 \%$ \\
Mid-esophagus & & \\
\hline Histology & & $10 \%$ \\
Adenocarcinoma & 36 & $90 \%$ \\
Squamous cell carcinoma & 4 & $15 \%$ \\
\hline Clinical Stage & & \\
T4N0 & & \\
T3N1 & & \\
T3N0 & & \\
T2N1 & & \\
\hline
\end{tabular}


Table 2

Dose-Limiting Toxicity By Level

\begin{tabular}{|l|l|l|l|l|}
\hline Level & Capecitabine & $\mathbf{N}$ & Dose-limiting Toxicity & \% DLT \\
\hline I & $500 \mathrm{mg}$ & 7 & One grade 3 dysphagia & $14.3 \%$ \\
\hline II & $1000 \mathrm{mg}$ & 3 & None & $0 \%$ \\
\hline III & $1500 \mathrm{mg}$ & 6 & One grade 3 dysphagia & $16.7 \%$ \\
\hline IV & $2000 \mathrm{mg}$ & 3 & None & $0 \%$ \\
\hline V & $2500 \mathrm{mg}$ & 6 & One thrombocytopenia & $16.7 \%$ \\
\hline VI & $3000 \mathrm{mg}$ & 6 & One fatigue & $16.7 \%$ \\
\hline VII & $3500 \mathrm{mg}$ & 9 & $\begin{array}{l}\text { One grade 3 pneumonitis } \\
\text { One withdrawal for nausea } \\
\text { One withdrawal for neuropathy }\end{array}$ & $33.3 \%$ \\
\hline
\end{tabular}




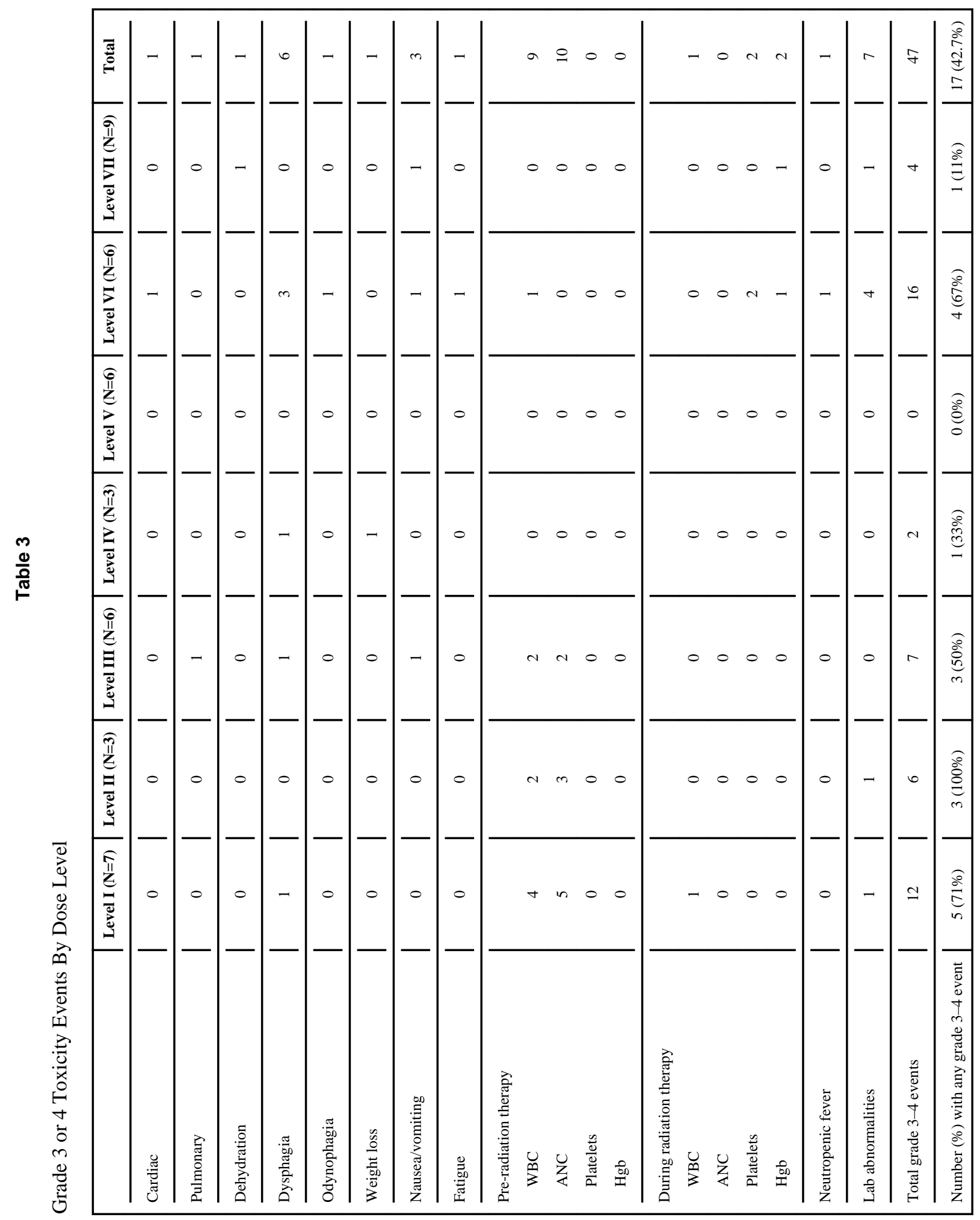

J Thorac Oncol. Author manuscript; available in PMC 2014 April 01. 


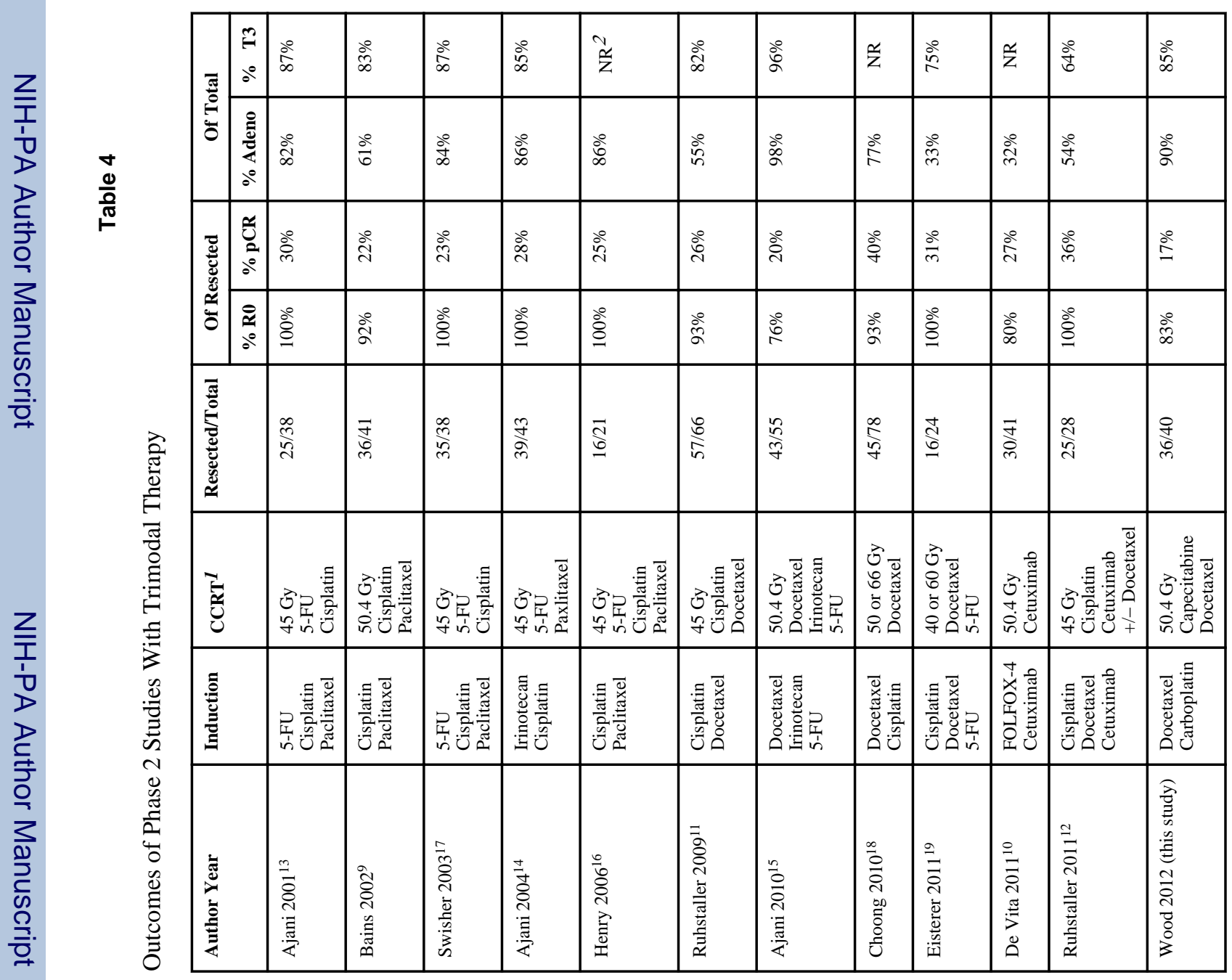


